\title{
Nanomaterials: A Primer
}

\author{
Matthew N. O. Sadiku ${ }^{1}$, Yogita P. Akhare ${ }^{1}$, Abayomi Ajayi-Majebi², and Sarhan M. Musa ${ }^{1}$ \\ ${ }^{1}$ Roy G. Perry College of Engineering \\ Prairie View A\&M University \\ Prairie View, TX 77446 \\ ${ }^{2}$ Department of Manufacturing Engineering \\ Central State University \\ P.O. Box 1004 \\ Wilberforce, OH 45384-1004
}

\begin{abstract}
Nanotechnology refers to the understanding and control of matter at dimensions between approximately 1 and 100 nm. Nanomaterials, the result of the manufacturing application of nanotechnology, are materials possessing, at minimum, one external dimension measuring 1-100nm. They are the building blocks of nanotechnology as they possess unique structures and properties that distinguish them from other materials. More and more nanomaterials are expected to be used in electronic and consumer products. This paper provides a primer on nanomaterials and their diverse applications.
\end{abstract}

Key Words: Nanomaterials, Nanotechnology.

\section{INTRODUCTION}

Techniques are now available, making it possible to manipulate materials on the atomic or molecular scale to produce objects that are no more than a few nanometres in diameter. The processes used to make and manipulate such materials are known as nanotechnology, the materials or objects themselves are called nanomaterials, and the study and discovery of these materials is known as Nanoscience [1,2].

Nanotechnology is the design, fabrication, and application of nanostructures or nanomaterials. It has emerged as a catalyst for revolutionary commercial applications.

It allows us to create functional materials, devices, and systems by controlling matters at the atomic and molecular scales. Nanotechnologies have provided enormous benefits in numerous new research areas and in applications such as material science, engineering, communication, electronics, medicine, energy, and the environment. They have also been used in the field of environmental management to improve air, water, and soil quality [3]. They can be used to improve products and solve global problems in areas such as energy, water, medicine, and environmental remediation. Significant fields of nanotechnology include nanomaterials, nanodevices, nanomanufacturing, and nano measurement. Nanomaterials may be regarded as the cornerstones of nanoscience and nanotechnology.

\section{WHAT IS NANOMATERIAL?}

A nanomaterial (NM) (or nano-sized materials) is the material with any external dimension in the nanoscale, i.e., one or more external dimensions is in the size range $1 \mathrm{~nm}-100 \mathrm{~nm}$. Thus, by definition, nanomaterials must have at least one dimension that is less than approximately 100 nanometers. Nanomaterials are of interest because at this scale unique optical, magnetic, electrical, thermo-physical, or mechanical properties. These emergent properties have great potential applications in electronics, medicine, and other fields. Materials with structure at the nanoscale often 
have unique optical and electronic properties. As shown in Figure 1, nanomaterials may be classified as Zero dimensional, one dimensional, two dimensional, three-dimensional nanostructures [4].

Nanomaterials can occur in many ways: natural, incidental, or manufactured. That is, they can exist naturally, as the by-products of an industrial process, or be produced through some engineering process. Nanoscale materials can be engineered from minerals and nearly any chemical substance. Engineered nanomaterials have been deliberately manufactured by humans to have certain required properties. Nanomaterials may also be produced incidentally as a by-product of mechanical or industrial processes. Natural nanomaterials can be produced by biological systems [5].

\section{APPLICATIONS}

Nanomaterials are chemical substances or materials that are manufactured and used on a tiny scale. They exhibit desirable and useful properties. They are used in various applications such as in medicine or healthcare, electronic devices, sunscreens, environmental protection, products including paints, filters, insulation, and lubricant additives, etc. Some of these do not affect the environment, while others affect it. We consider the following typical applications [6,7].

- Healthcare: The healthcare field uses nanomaterials in a variety of ways. One major use being drug delivery. Nanoparticles can be developed to assist the transportation of chemotherapy drugs directly to cancerous growths. Nanomaterials are increasingly used in biomedical applications (as therapeutics and as diagnostic tools). However, before NMs can be safely applied in a clinical setting, their toxicity should be carefully assessed. Currently, little is known about the toxicity of nanomaterials. A variety of dental NMs is commercially available.

- Environmental Improvement: Engineered nanomaterials may be released into the environment through industrial and environmental applications. The power of engineered nanomaterials can be harnessed to benefit the environment and improve public health. Nanomaterials can also be used in environmental remediation or clean-up to bind with and neutralize toxins. Environmental preservation processes make use of nanomaterials to play a role in the treatment of polluted water.

- Industries: The use of nanomaterials is prevalent in a wide range of industries and consumer products. In the cosmetics industry, mineral nanoparticles -such as titanium oxide -are used in sunscreen. The sports industry has been using antimicrobial nanotechnology in items such as the towels and mats used by sports professionals to prevent illnesses caused by bacteria. Nanomaterials have contributed significantly to major advances in electronics, leading to faster, smaller, and more portable systems that can manage and store larger amounts of information. They are also used in the computing and textile industries.

- Microelectronics Sector: This sector has paid special attention to miniaturization, which involves reducing the size of circuits like transistors, capacitors, and resistors. Miniaturization enables microprocessors developed using these parts to operate much faster. However, there are a number of technical challenges to achieving these advancements, such as the lack of ultrafine precursors to make these parts, inadequate dissipation of huge amounts of heat generated by these microprocessors, and poor reliability) etc. Nanomaterials help overcome these barriers by offering manufacturers materials with better thermal conductivity, nanocrystalline starting materials, ultra-high-purity materials, and longer-lasting, durable interconnections.

- Energy Sector: Nanotechnology is finding application in traditional energy sources and enhancing alternative energy approaches to help meet the world's increasing energy demands. The use of nanomaterials is beneficial to the energy sector in that they can make the existing methods of generating energy - such as solar panels - more efficient and cost-effective, as well as opening up new ways in which to both harness and store energy.

- Consumer Products: Nanomaterials are increasingly employed in new and existing consumer products. Like all products, nano-enabled products have stages in their lifecycle of use where they can encounter humanmade or environmental factors that may lead them to transform physically and/or chemically from their original form. Nanomaterials in consumer products are illustrated in Figure 2 [8]. 
- Food Industry: Nanotechnology is revolutionizing the food industry. As shown in Figure 3, potential applications of nanotechnology in the food chain include food storage, food quality control, and food packaging [9]. Nanomaterials are increasingly being added to food handling and packaging materials. Nano sensors composed of different nanomaterials have been used for the detection of pesticides, fertilizers, and other toxic chemicals present in various foodstuffs.

Other nanomaterials applications include military applications, photovoltaic cells, paints, catalysts, manufacturing processes, spacecraft, and wastewater management. Some of the applications of NMs are illustrated in Figure 4 [10].

\section{BENEFITS AND CHALLENGES}

Nanotechnology has attracted a great deal of interest because of the needs and applications of nanomaterials in many areas of human endeavors, including industry, agriculture, engineering, business, medicine, and public health. Nanomaterials (NMs) have been the object of numerous public and private initiatives to manage their risks and maximize their benefits. They possess unique structures and properties that distinguish them from other materials. NMs are of interest due to their unique optical, magnetic, electrical, and other properties at this scale. These emergent properties have great potential applications in sports equipment, sun cream, medicine, and consumer products such as cosmetics, paints, fabrics, and electronics. NMs have some advantages over their bulk counterparts due to their tunable physical, chemical, and biological properties with the enhanced performance [11].

Although engineered nanomaterials provide great benefits, we know very little about the potential effects on human health and the environment. NMs are yet to be thoroughly researched as to their potential health, safety, and environmental risk to human life. In some workplaces, workers using nanotechnology in research or production may be exposed to nanomaterials through inhalation, skin contact, or ingestion. Some have expressed concern that nanomaterials such as carbon nanotubes and nanofibers may cause detrimental pulmonary effects. Accumulation of nanomaterials over time in other aspects of the environment, such as soils - through sewage sludge - is an additional concern. There is no means of detecting levels of nanoparticles in the air-a particular concern in workplaces where nanomaterials are being used. Because nanotechnology is a recent development, there is no regulation on the occupational exposure limit of nanomaterials in workplaces [12,13]. The risk associated with NMs has always been a concern for investors in their decision-making process.

\section{CONCLUSION}

Nanomaterials are partially characterized by their tiny size, measured in nanometers. Applications of nanotechnology and nanomaterials are delivering in both expected and unexpected ways on nanotechnology's promise to benefit society.

Nanomaterials are gradually becoming commercialized and emerging as commodities. Because nanotechnology is a recent development, the health and safety effects of exposures to nanomaterials are subjects of ongoing research. Since nanomaterials are increasingly a part of everyday consumer products, it is expedient and imperative that both workers and end-users be protected from inhalation of potentially toxic NPs.

For more information about nanomaterials, one should consult the books in [14-23] and the related journals:

- Nanomaterials

- Journal of Nanomaterials

- American Journal of Nanomaterials

- Journal of Nanomaterials \& Molecular Nanotechnology.

- Journal of Nanotechnology and Nanomaterials 


\section{REFERENCES}

[1] M. N. O. Sadiku, M. Tembely, and S.M. Musa," Nanotechnology: an Introduction," International Journal of Software and Hardware Research in Engineering, vol. 4, no. 5, May. 2016, pp. 40-44.

[2] M. N. O. Sadiku, S. M. Musa, and O. S. Musa, "Nanotechnology in Chemical Industry," Invention Journal of Research Technology in Engineering and Management, vol. 2, no. 1, Jan. 2018, pp. 24-26.

[3] Z. Chen et al., "A review of environmental effects and management of nanomaterials, Toxicological \& Environmental Chemistry, vol. 93, no. 6, 2011, pp. 1227-1250.

[4] A. Alagarasi, "Chapter - Introduction to nanomaterials," December 2011, file:///C:/Users/12818/Downloads/IntroductiontoNanomaterials\%20(1).pdf

[5] "Nanomaterials," Wikipedia, the free encyclopedia, https://en.wikipedia.org/wiki/Nanomaterials

[6] A. S. Ali, "Application of nanomaterials in environmental improvement," in Nanotechnology and the Environment. Intechopen, 2020. Also available online:

https://www.intechopen.com/books/nanotechnology-and-the-environment/application-of-nanomaterials-inenvironmental-improvement

[7] "What is a nanomaterial? - Definition, examples and uses," https://www.twi-global.com/technical-knowledge/faqs/what-is-a-nanomaterial

[8] "Nanomaterials in consumer products," https://www.nist.gov/programs-projects/nanomaterials-consumer-products

[9] M. Shafiq et al., "An overview of the applications of nanomaterials and nanodevices in the food industry," Foods, vol. 9, no. 2, February 2020.

[10] E. Moore," Nanomaterials: To use or not to use Breadcrumb," September 2019, https://www.rit.edu/spotlights/nanomaterials-use-or-not-use

[11]P. C. Ray, H. Yu, and P. P. Fu, "Toxicity and environmental risks of nanomaterials: challenges and future needs," Journal of Environmental Science and Health Part C, vol. 27, no. 1, 2009, pp. 1-35.

[12] "Working safely with nanomaterials," https://www.osha.gov/Publications/OSHA_FS-3634.pdf

[13] T. A. Hemphill, "Regulating nanomaterials: A case for hybrid governance," Bulletin of Science, Technology \& Society, vol. 36, no. 4, 2016, pp. 219-228.

[14] G. A. Ozin and A. Arsenault, Nanochemistry: A Chemical Approach to Nanomaterials. RSC Publishing, 2nd edition, 2015.

[15] Y Gogotsi (ed.), Nanomaterials Handbook. Boca Raton, FL: CRC Press, 2nd edition, 2017.

[16] C. Bréchignac, and P. Houdy, and M. Lahmani (eds.), Nanomaterials and Nanochemistry. Springer, 2008.

[17] C. N. R. Rao, A. Muller, and A. K. Cheetham (eds.), The Chemistry of Nanomaterials. Wiley- VCH Verlag, 2004.

[18] Y. Gogotsi and V. Presser (eds.), Carbon Nanomaterials. Boca Raton, FL: CRC Press, 2013.

[19] D. M. Ashby, P. Ferreira, D. L. Schodek, Nanomaterials, Nanotechnologies and Design: an Introduction to Engineers and Architects. Butterworth-Heinemann, 2009.

[20] A. Amrane et al.(eds.), Nanomaterials for Air Remediation. Elsevier, 2020.

[21] C. S. S. R. Kumar (ed.), Magnetic Nanomaterials. John Wiley \& Sons, 2009.

[22] T. Awan, A. Bashir, and A.Tehseen, Chemistry of Nanomaterials: Fundamentals and Applications. Elsevier, 2020.

[23] J. H. Kiat (ed.), Nanomaterials in Energy Devices. Boca Raton, FL: CRC Press, 2017.

\section{ABOUT THE AUTHORS}

Matthew N.O. Sadiku is a professor emeritus in the Department of Electrical and Computer Engineering at Prairie View A\&M University, Prairie View, Texas. He is the author of several books and papers. His areas of research interest include computational electromagnetics and computer networks. He is a fellow of IEEE.

Yogita P. Akhare is a doctoral student at Prairie View A\&M University, Prairie View, Texas. Her research interests include machine drives and nanotechnology. yakhare@student.pvamu.edu

Abayomi Ajayi-Majebi is a professor in the Department of Manufacturing Engineering at Central State University in Wilberforce, Ohio. In 2015 he was honored by the White House as a Champion of Change for his significant contributions to the engineering education of minority students. He is a senior member of both the Society of Manufacturing Engineers and the American Society for Quality.

Sarhan M. Musa is a professor in the Department of Electrical and Computer Engineering at Prairie View A\&M University, Texas. He has been the director of Prairie View Networking Academy, Texas, since 2004. He is an LTD Sprint and Boeing Welliver Fellow. His areas of research interest include computational electromagnetics and computer networks. 
International Journal of Advances in Scientific Research and Engineering (ijasre), Vol 7 (3), March -2021

(a)

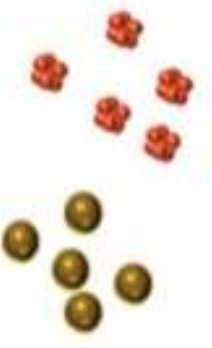

(b)

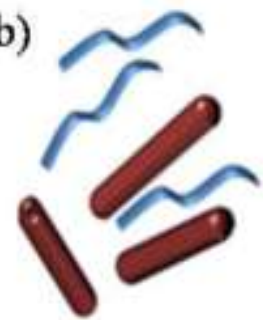

(c)

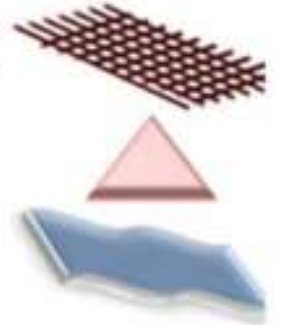

(d)

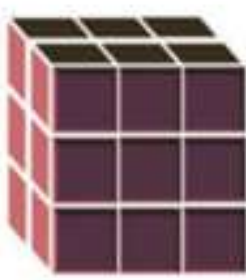

Figure 1: Classification of Nanomaterials (a) 0D spheres and clusters; (b) 1D nanofibers, nanowires, and nanorods; (c) 2D nanofilms, nanoplates, and networks; (d) 3D nanomaterials [4].

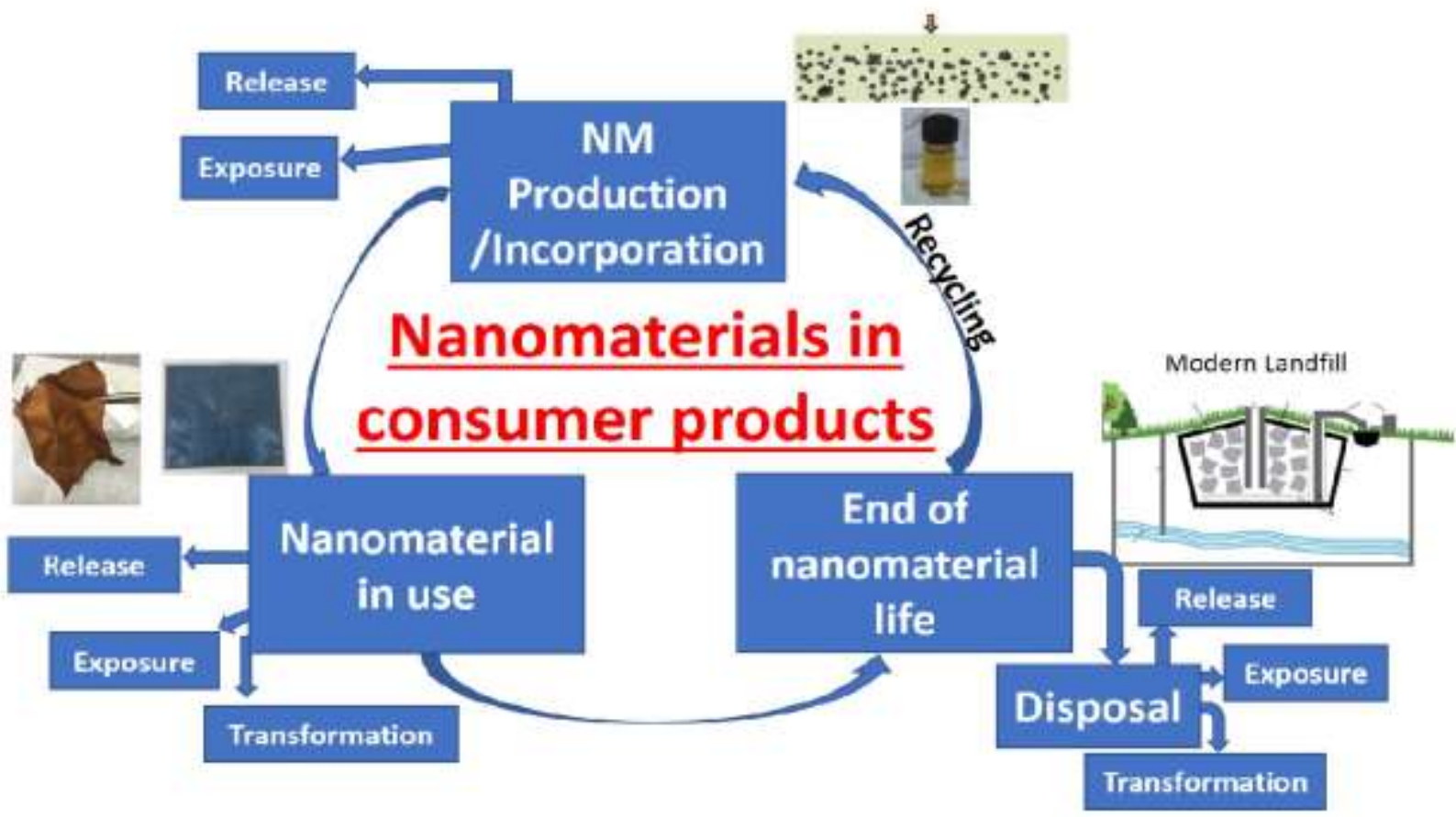

Figure 2: Nanomaterials in consumer products [8]. 
International Journal of Advances in Scientific Research and Engineering (ijasre), Vol 7 (3), March -2021

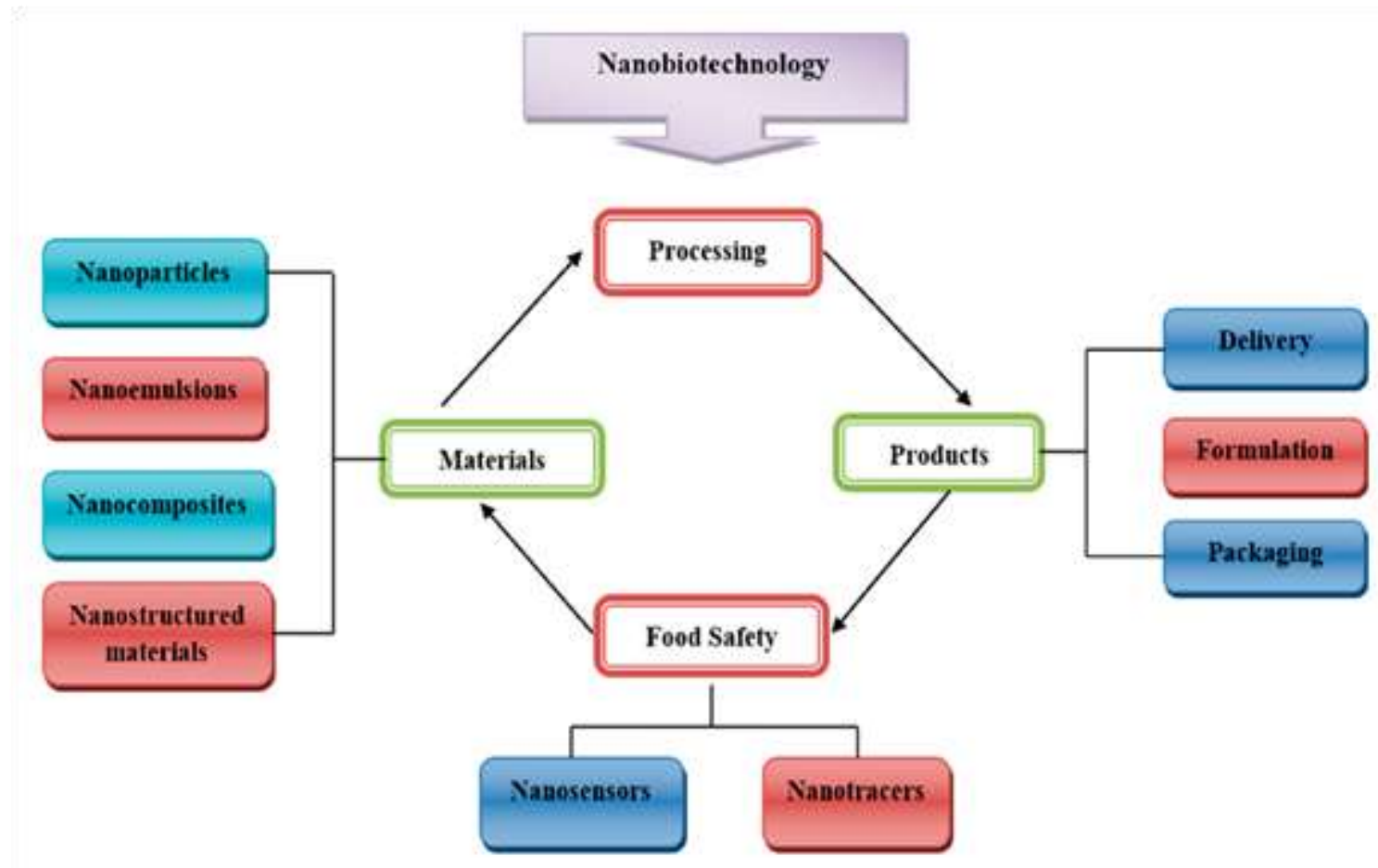

Figure 3: Nanotechnology in various sectors of the food industry [9].

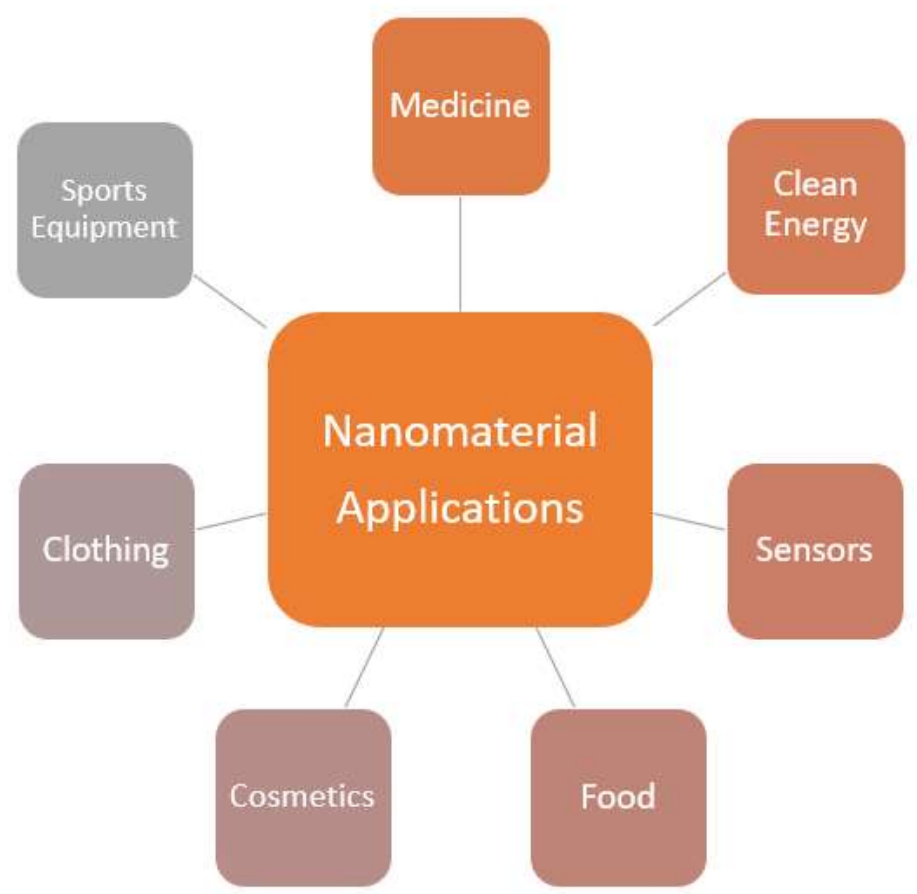

Figure 4: Some of the applications of NMs [10]. 